\title{
Three-field versus two-field lymph node dissection for thoracic esophageal squamous cell carcinoma: a propensity score- matched comparison
}

\author{
Longlong Shao ${ }^{1,2}$, Ting Ye ${ }^{1,2}$, Longfei $\mathrm{Ma}^{1,2}$, Dong $\mathrm{Lin}^{1,2}$, Hong $\mathrm{Hu}^{1,2}$, Yihua Sun ${ }^{1,2}$, Yawei Zhang ${ }^{1,2}$, Jiaqing \\ Xiang $^{1,2 \#}$, Haiquan Chen ${ }^{1,2 \#}$ \\ ${ }^{1}$ Department of Oncology, Shanghai Medical College, Fudan University, Shanghai 200032, China; ${ }^{2}$ Department of Thoracic Surgery, Fudan \\ University Shanghai Cancer Center, Shanghai 200032, China \\ \#These authors contributed equally to this work. \\ Correspondence to: Haiquan Chen. Department of Thoracic Surgery, Fudan University Shanghai Cancer Center, 270\# Dong'an Rd., Shanghai 200032, \\ China. Email: hqchen1@yahoo.com.
}

Background: For the surgery of esophageal cancer, cervical, mediastinal, and abdominal lymph node
dissection (three-field lymph node dissection, 3FLND) is still controversial in most countries. This study
aims to provide additional evidence of this procedure comparing with mediastinal-abdominal lymph node
dissection (two-field lymph node dissection, 2FLND) from a high volume center in China, and also attempts
to identify routes to reduce postoperative complications associated with 3FLND. Methods: From January 2009 to December 2013, 348 consecutive patients received esophagectomy with 3FLND and 1,406 patients received Ivor-Lewis with 2FLND in Fudan University Shanghai Cancer Center. After propensity-score matching, 282 pairs of cases without neoadjuvant treatment were selected. Postoperative outcomes and survival of the two groups were analyzed.

Results: Operative morbidity and mortality rates were $34.8 \%$ and $0.4 \%$ for 3FLND group; and $25.5 \%$ and $0.7 \%$ for 2FLND group. Compared with the 2FLND group, the 3FLND group reported more cases of anastomotic leakage $(14.9 \%$ vs. $4.3 \%, \mathrm{P}<0.001)$. Multivariate analysis showed that an independent factor of anastomotic leakage was the anastomotic location $[\mathrm{HR}=0.096(0.037-0.247), \mathrm{P}<0.001]$ rather than the extent of lymph node dissection. The intrathoracic anastomosis subgroup of 3FLND showed a similar leakage rate as the 2 FLND group ( $4.2 \%$ vs. $4.3 \%$ ). At a median follow-up of 42 months, no significant difference was observed in overall survival between the groups $(\mathrm{P}=0.529)$. A subgroup of patients with N1 status who underwent 3FLND showed a better survival trend than those who underwent 2FLND ( $\mathrm{P}=0.093$ ). No significant difference was observed in overall survival between the intrathoracic anastomosis subgroup and cervical anastomosis subgroup $(\mathrm{P}=0.334)$.

Conclusions: Intrathoracic anastomosis may reduce the incidence of anastomotic leakage in esophagectomy with 3FLND, with no compromise on overall survival. Compared with the 2FLND, patients with N1 status might benefit more from the 3FLND technique.

Keywords: Esophageal squamous cell carcinoma (ESCC); three-field lymph node dissection (3FLND); two-field lymph node dissection (2FLND)

Submitted Feb 12, 2018. Accepted for publication May 03, 2018.

doi: $10.21037 /$ jtd.2018.05.69

View this article at: http://dx.doi.org/10.21037/jtd.2018.05.69 


\section{Introduction}

Esophagectomy with three-field lymph node dissection (3FLND) has been proposed for decades, and now being the main surgical procedure for esophageal cancer in Japan. Although many studies have confirmed its survival benefit $(1,2), 3$ FLND is not widely used in other countries because it is associated with greater trauma and more complications. Even in china, where the upper and middle thoracic esophageal squamous cell carcinoma (ESCC) accounted for a large proportion, the procedure of 3FLND was not widely applied. Studies comparing 3FLND and two-field lymph node dissection (2FLND) reported by Japanese scholars lack evidence from other countries and large sample sizes (3).

Since the year 2000, esophagectomy with 3FLND has been the routine surgical treatment for esophageal cancer in our center. In this study, we retrospectively analyzed postoperative outcomes and survival of patients who have undergone 3FLND and compared them with those of patients who have undergone the Ivor-Lewis procedure, which is a classical procedure involving 2FLND. We aim to provide clinical evidence to support the use of 3FLND and perhaps find a modified version of this technique.

\section{Methods}

From January 2009 to December 2013, 3,062 patients received esophagectomy in the department of thoracic surgery in Fudan University Cancer Center, and 2,672 patients of them were diagnosed with squamous cell carcinoma. Of these, 124 patients received neoadjuvant therapy, accounting for only a small part, were excluded. Only patients who underwent esophagectomy with 3FLND and Ivor-Lewis were enrolled (Figure 1). The surgical technique was chosen by thoracic surgeons based on the results of preoperative examinations, including thoracoabdominal enhanced computed tomography, cervical ultrasonography, esophagoscopy, and positron emission tomography, if necessary. Patients with any of the three following conditions, underwent 3FLND as a priority in line with evidence reported previously (4,5): (I) tumor involving the upper thoracic esophagus; (II) enlarged and resectable cervical recurrent laryngeal nerve lymph nodes or supraclavicular lymph nodes; (III) suspicious of lymph nodes metastasis adjacent to the thoracic recurrent laryngeal nerves.

\section{Surgical procedure}

Esophagectomy with 3FLND was performed via a right muscle sparing thoracotomy, followed by upper midline laparotomy and cervical collar incision consisting of the following three steps: (I) the esophagus was dissected from the diaphragm to the apex of the chest, along with the mediastinal and hilar lymph nodes dissected, including the lymph nodes adjacent to the bilateral recurrent laryngeal nerves. The azygos vein arch was cut off and the thoracic duct was ligated regularly; (II) during the laparotomy, the stomach was mobilized with reserve of the right gastroepiploic artery, and lymph nodes along the hepatic artery and the celiac axis were resected. Meanwhile, both

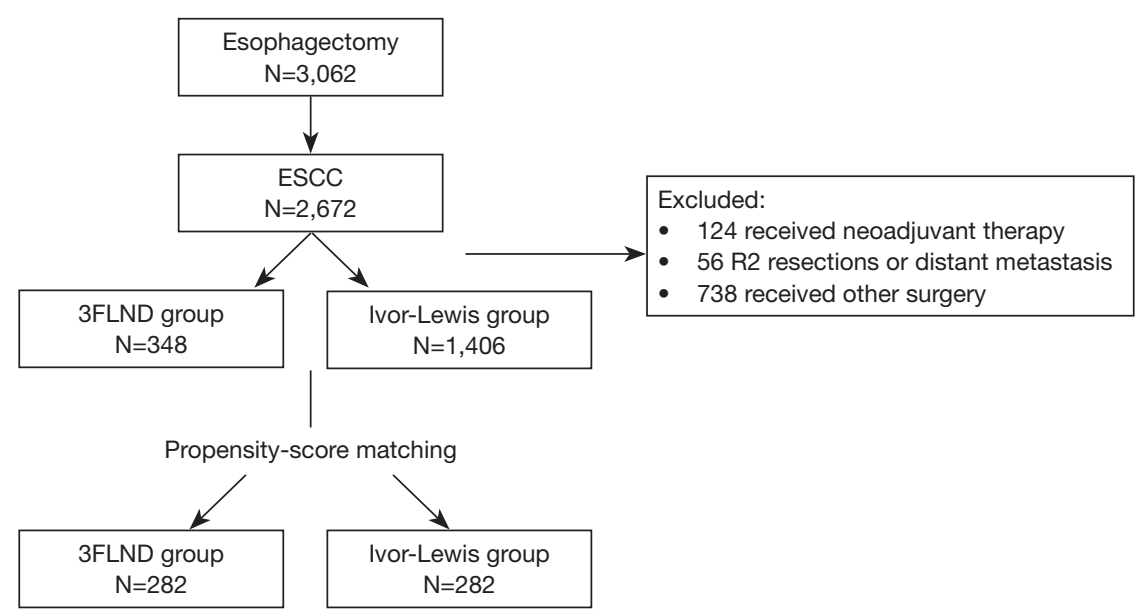

Figure 1 Flow diagram of this study. ESCC, esophageal squamous cell carcinoma; 3FLND, three-field lymph node dissection. 
sides of the cervical recurrent laryngeal nerve lymph nodes and the supraclavicular lymph nodes were dissected through a cervical incision; (III) the gastric tube was pulled to the left-side of the neck through the post-mediastinal route and an end-to-side anastomosis was constructed. For patients receiving intrathoracic anastomosis, the surgery began with the cervical lymph node dissection and gastric mobilization, and ended with the thoracotomy and mechanical anastomosis in the right thoracic apex.

In Ivor-Lewis group, instead of cervical lymphadenectomy, upper mediastinal lymph node dissection was performed. The anastomosis in this group was performed via circular stapling in the right thoracic apex.

\section{Follow-up}

Patients were asked to review in the clinic every three months in the first 2 years, and every 6 months in the following years. Follow-up investigations included clinical examinations, a thoracic CT scan, cervical and abdominal ultrasonography, barium swallow test, esophagoscopy, and sometimes positron emission tomography. Survival data were investigated from clinic records or telephone interviews.

\section{Statistical analysis}

The clinical database analyzed for this study was approved by the institutional review board of Fudan University Shanghai Cancer Center. Clinical data from the patients were reviewed retrospectively. Clinicopathologic characteristics and postoperative profiles were analyzed and compared. To minimize the selection bias in this retrospective study, we performed propensity score matching via multivariable logistic regression for each patient, using variables such as age, gender, body mass index, comorbidities, tumor location, and pTNM stage. Propensity scores were computed as the conditional probability of receiving either $3 \mathrm{FLND}$ or $2 \mathrm{FLND}$, using the nearest neighbor-matching algorithm. We created propensity score-matched pairs by a 1:1 match. The caliper definition was set at 0.01 . Eventually, 282 paired cases were matched from the cohort, and the two groups were comparable in terms of patient characteristics.

Survival rate was calculated by the Kaplan-Meier method. The significance of differences in survival was evaluated by the log rank test. Cox regression hazard model was used for univariate and multivariate analysis to assess the independent influence of variables on overall survival. Two-sided $\mathrm{P}$ values of less than 0.05 were considered statistically significant. All analyses were performed by using SPSS (version 19.0) for Windows of IBM Corporation.

\section{Results}

\section{Basic characteristics}

Of the 1,810 identified patients who finally underwent either 3FLND or Ivor-Lewis with 2FLND, 56 patients with non-radical resection ( $\mathrm{R} 2$ resections or distant metastasis) were excluded, leaving 1,754 patients for final analysis. Characteristics of these patients are presented in Table 1. Before matching, the 3FLND group had more patients with tumor located in upper thoracic esophagus and with stage III than 2 FLND group, $17.8 \%$ vs. $2.8 \%, 60.6 \%$ vs. $44.0 \%$, respectively. The 3FLND group also had more patients with digestive complications, such as cirrhosis, a history of esophageal disease or a history of upper gastrointestinal surgery. After matching, these factors were balanced and the groups were comparable.

\section{Operative and postoperative features}

We performed cervical lymphadenectomy during the abdominal surgery; thus the 2FLND group, the surgical duration was similar in both groups. Patients in The two groups also had similar blood loss and postoperative length of hospital stay. More lymph nodes were harvested in 3 FLND group (38.5 vs. 23.1, $\mathrm{P}<0.001$ ), and more metastatic lymph nodes were found in this group (3.5 vs. 1.6, $\mathrm{P}<0.001)$. More surgical complications occurred in the 3 FLND group ( $34.8 \%$ vs. $25.5 \%, \mathrm{P}=0.017)$, especially anastomotic leakage $(14.9 \%$ vs. $4.3 \%, \mathrm{P}<0.001)$. In all, 10 patients underwent reoperation because of major complications: 1 acute peritonitis, 3 thoracic hemorrhage, 1 chylothorax and 1 esophagotracheal fistula in the 3FLND group; 1 intestinal obstruction, 2 thoracic hemorrhage, and 1 esophagotracheal fistula in the 2FLND group. The patient with esophagotracheal fistula in the 3FLND group experienced additional complications of respiratory failure and renal failure, and died within one month of the surgery. The other two deaths in the 2FLND group were due to anastomotic leakage and respiratory failure which occurred within 2 and 4 months after the surgery, respectively. Operative and postoperative features are listed in Table 2. 
Table 1 Patients characteristics

\begin{tabular}{|c|c|c|c|c|c|c|}
\hline Characteristics & \multicolumn{3}{|c|}{ Before matching } & \multicolumn{3}{|c|}{ After matching } \\
\hline Age & $60 \pm 7$ & $60 \pm 8$ & 0.823 & $60 \pm 7$ & $60 \pm 6$ & 0.875 \\
\hline Gender (M/F) & $292 / 56$ & $1,159 / 247$ & 0.512 & $238 / 44$ & $238 / 44$ & 1.000 \\
\hline Comorbidities, n (\%) & & & $<0.001$ & & & 0.441 \\
\hline Hypertension & $38(10.9)$ & $208(14.8)$ & & $31(11.0)$ & $33(11.7)$ & \\
\hline Diabetes & $13(3.7)$ & $52(3.7)$ & & $10(3.5)$ & $16(5.7)$ & \\
\hline Respiratory diseases & $9(2.6)$ & $14(1.0)$ & & $4(1.4)$ & $5(1.8)$ & \\
\hline Digestive diseases & $47(13.5)$ & $27(1.9)$ & & $21(7.4)$ & $14(5.0)$ & \\
\hline I & $26(7.5)$ & $92(6.5)$ & & $25(8.9)$ & $26(9.2)$ & \\
\hline II & $111(31.9)$ & $695(49.4)$ & & $97(34.4)$ & $86(30.5)$ & \\
\hline III & $211(60.6)$ & $619(44.0)$ & & $160(56.7)$ & $170(60.3)$ & \\
\hline Location, n (\%) & & & $<0.001$ & & & 0.126 \\
\hline Upper & $62(17.8)$ & $39(2.8)$ & & $25(8.9)$ & $15(5.3)$ & \\
\hline Middle & $230(66.1)$ & $933(66.4)$ & & $201(71.3)$ & $220(78.0)$ & \\
\hline Lower & $56(16.1)$ & $434(30.9)$ & & $56(19.9)$ & $47(16.7)$ & \\
\hline
\end{tabular}

*, 7th edition of AJCC Cancer Staging Manual. Cervical lymph node metastasis defined as lymph node metastasis rather than distant metastasis. 3FLND, three-field lymph node dissection; 2FLND, two-field lymph node dissection.

\section{Survival}

After a median follow-up period of 42 months, 250 patients from the 3FLND group and 226 from the 2FLND group provided survival information. No significant difference was found in overall survival between the two groups (Figure 2). The median OS was 52 months in the 3FLND group and 54 months in $2 \mathrm{FLND}$ patients $(\mathrm{P}=0.529)$. Threeyear OS rates of the 3 FLND group and the 2 FLND group were $60.8 \%$ and $64.6 \%$, while 5 -year OS rates were $48 \%$ and $52.6 \%$, respectively.

In the univariate analysis, the age, gender, tumor localization, and extended lymph node dissection did not have a significant influence on overall survival (Table 3). Nodal status and $\mathrm{T}$ stage were both independent factors. In the multivariable analysis, nodal status was the only independent factor.

No statistical difference was found in survival according to pathologic stage or $\mathrm{N}$ stage between the two groups (Figures 3,4). For the subgroup of patients with N1 status, those who underwent 3FLND seemed to have a better survival rate than those who underwent $2 \mathrm{FLND}(\mathrm{P}=0.093)$.

\section{Discussion}

Surgeons have gradually accepted the 3FLND technique since its emergence in the 1980s $(1,6)$. Research shows that it has the advantages of survival benefits and disadvantages of high incidence of complications, of which anastomotic fistula is a typical one. The anastomosis in 3FLND is performed in neck conventionally, and the reported leakage rate is $15.8-38.6 \%(7,8)$. In case of anastomotic leakage, patients may experience additional complications, which may be serious and life-threatening. However, the reported incidence rate of anastomotic fistula is much lower in another common type of surgery for esophageal 
Table 2 Operative and postoperative features

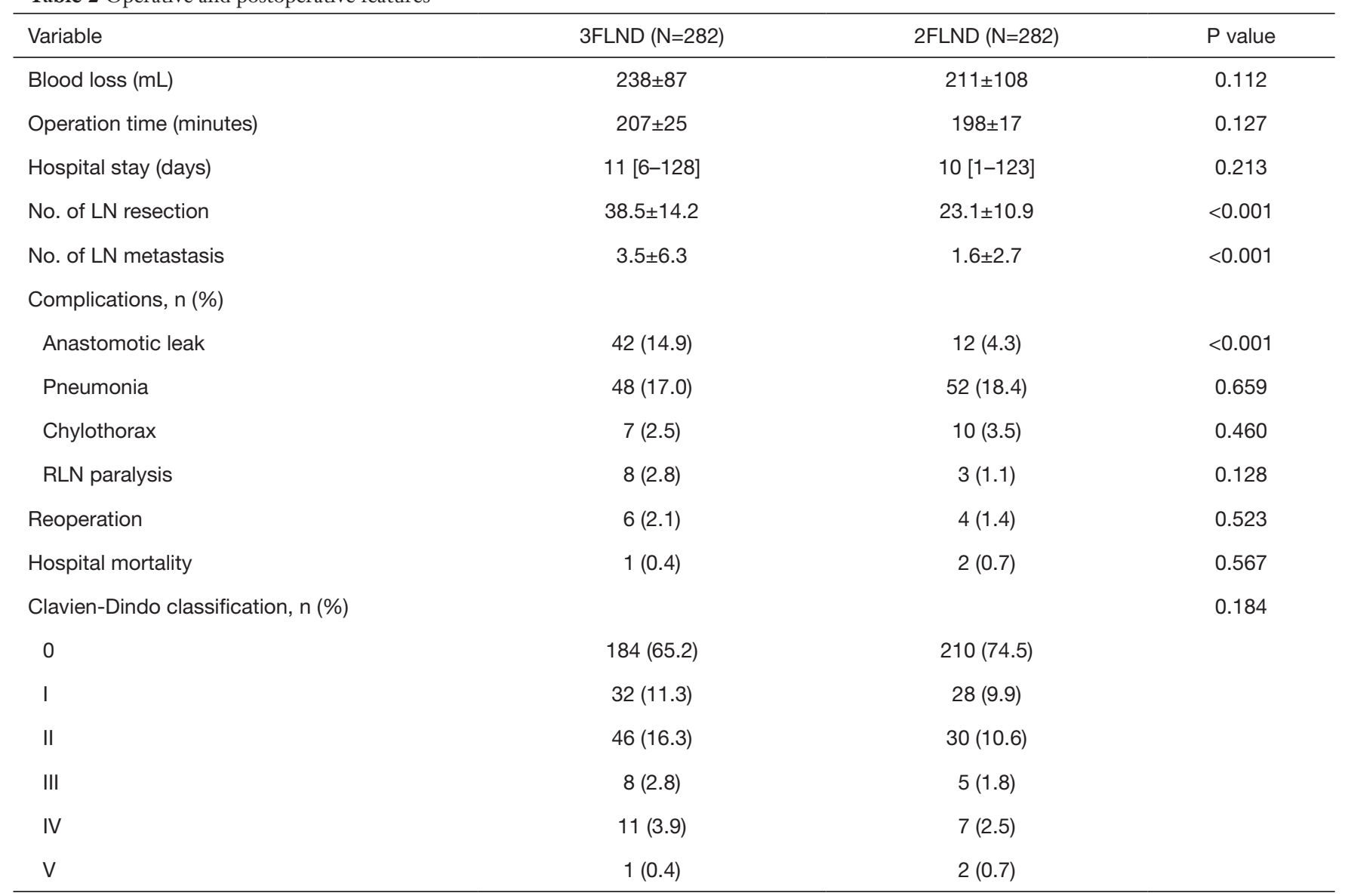

3FLND, three-field lymph node dissection; 2FLND, two-field lymph node dissection; LN, lymph node; RLN, recurrent laryngeal nerve.

cancer: the Ivor-Lewis technique $(3.5-5.1 \%)(9,10)$. Thus, we hypothesized that if the anastomosis is performed in the thoracic apex, with a safe resection margin, the incidence of anastomotic leakage in 3FLND may reduce. In both logistic and propensity score models (Table 4), we found that the application of intrathoracic anastomosis significantly reduced the risk of anastomotic leakage: from $25.7 \%(36 / 140)$ in the neck to $4.2 \%(6 / 142)$ in the thorax. The difference with the traditional I-L is that anastomosis in our 3FLND surgery located at the thoracic apex. We speculate that when the anastomosis is performed above the entrance to the thorax, it may be compressed by the sternum, leading to the lack of blood supply from the right gastroepiploic artery, which can be avoided by performing intrathoracic anastomosis.

Surgeons prefer cervical anastomosis to intrathoracic anastomotic, because management of cervical leakage is much easier while intrathoracic anastomotic fistula is more dangerous. We routinely placed a mediastinal drainage tube near the anastomosis until the patient resumed eating, whereby the mediastinum drainage patency could be maintained if an anastomotic fistula occurred. The risk of death due to intrathoracic fistula was not found to be high in this study or previous one (11). Another concern with intrathoracic anastomosis is that this procedure may increase local recurrence because of the short upper resection margin. In this study, a subgroup analysis showed intrathoracic anastomosis did not reduce the patients' postoperative survival (Figure 5). For esophageal cancer located above the aortic arch, we recommend careful consideration before opting for intrathoracic anastomosis. If the tumor is too close to the cutting edge, cervical anastomosis should be the primary choice.

Previous studies comparing 2FLND and 3FLND have shown that patients may benefit from 3FLND, especially those with positive lymph nodes $(3,12)$. However, this 


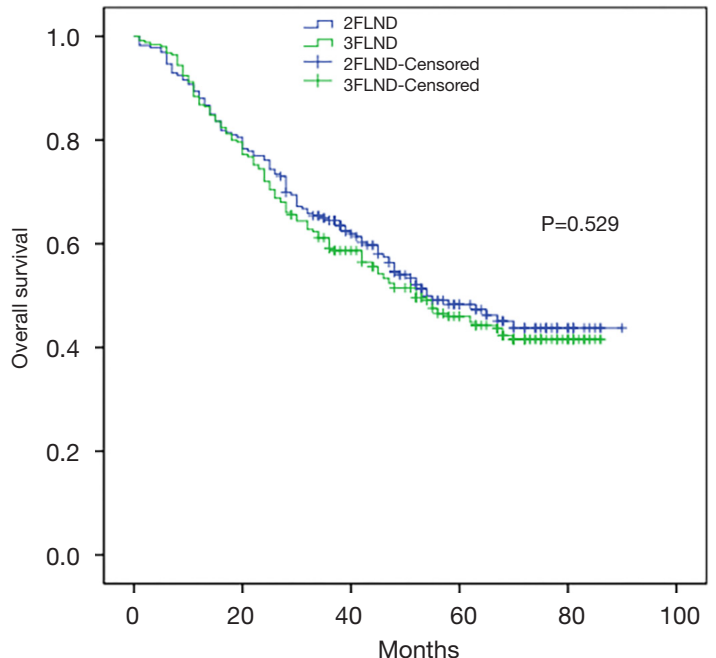

Figure 2 Kaplan-Meier curves for overall survival according to lymph node dissection. 2FLND, two-field lymph node dissection; 3FLND, three-field lymph node dissection.

Table 3 Univariate analyses of overall survival

\begin{tabular}{|c|c|c|c|}
\hline \multirow{2}{*}{ Variables } & \multicolumn{2}{|c|}{ Overall survival } & \multirow{2}{*}{$P$ value } \\
\hline & HR & $95 \% \mathrm{Cl}$ & \\
\hline \multicolumn{4}{|l|}{ Age } \\
\hline$\leq 60$ vs. $>60$ years & 0.819 & $0.636-1.054$ & 0.121 \\
\hline \multicolumn{4}{|l|}{ Gender } \\
\hline Male vs. female & 1.458 & $0.984-2.161$ & 0.060 \\
\hline \multicolumn{4}{|l|}{$\begin{array}{l}\text { Tumor location } \\
\text { (upper reference) }\end{array}$} \\
\hline Middle & 0.933 & $0.575-1.515$ & 0.780 \\
\hline Lower & 1.309 & $0.766-2.237$ & 0.326 \\
\hline \multicolumn{4}{|c|}{ N stage ( $p$ N0 reference) } \\
\hline N1 & 2.143 & $1.527-3.008$ & $<0.001$ \\
\hline N2 & 3.035 & $2.128-4.327$ & $<0.001$ \\
\hline N3 & 4.456 & $3.100-6.404$ & $<0.001$ \\
\hline \multicolumn{4}{|c|}{ T stage ( $\mathrm{p}$ T1 reference) } \\
\hline $\mathrm{T} 2$ & 2.431 & $1.429-4.135$ & 0.001 \\
\hline T3 & 3.136 & $1.919-5.126$ & $<0.001$ \\
\hline T4 & 3.540 & $2.004-6.252$ & $<0.001$ \\
\hline
\end{tabular}

Lymph node dissection

3FLND vs. 2FLND

$1.083 \quad 0.843-1.392 \quad 0.532$

3FLND, three-field lymph node dissection; 2FLND, two-field lymph node dissection.
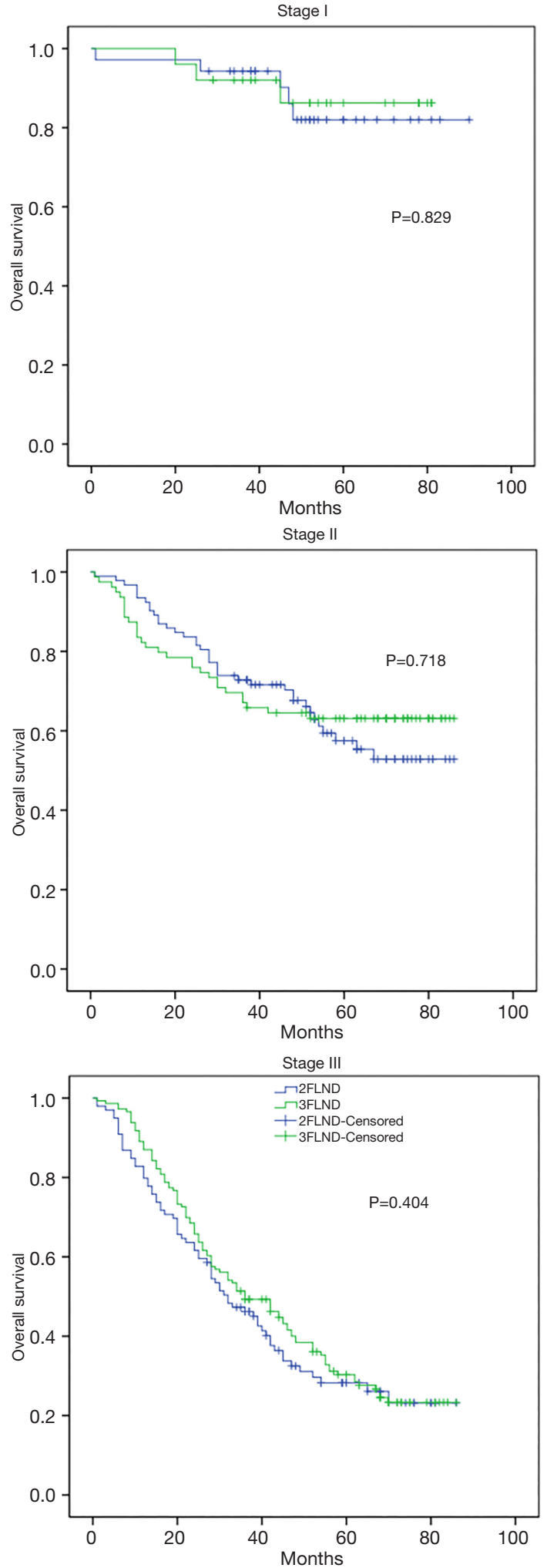

Figure 3 Kaplan-Meier curves for overall survival for each stage. 2FLND, two-field lymph node dissection; 3FLND, three-field lymph node dissection. 

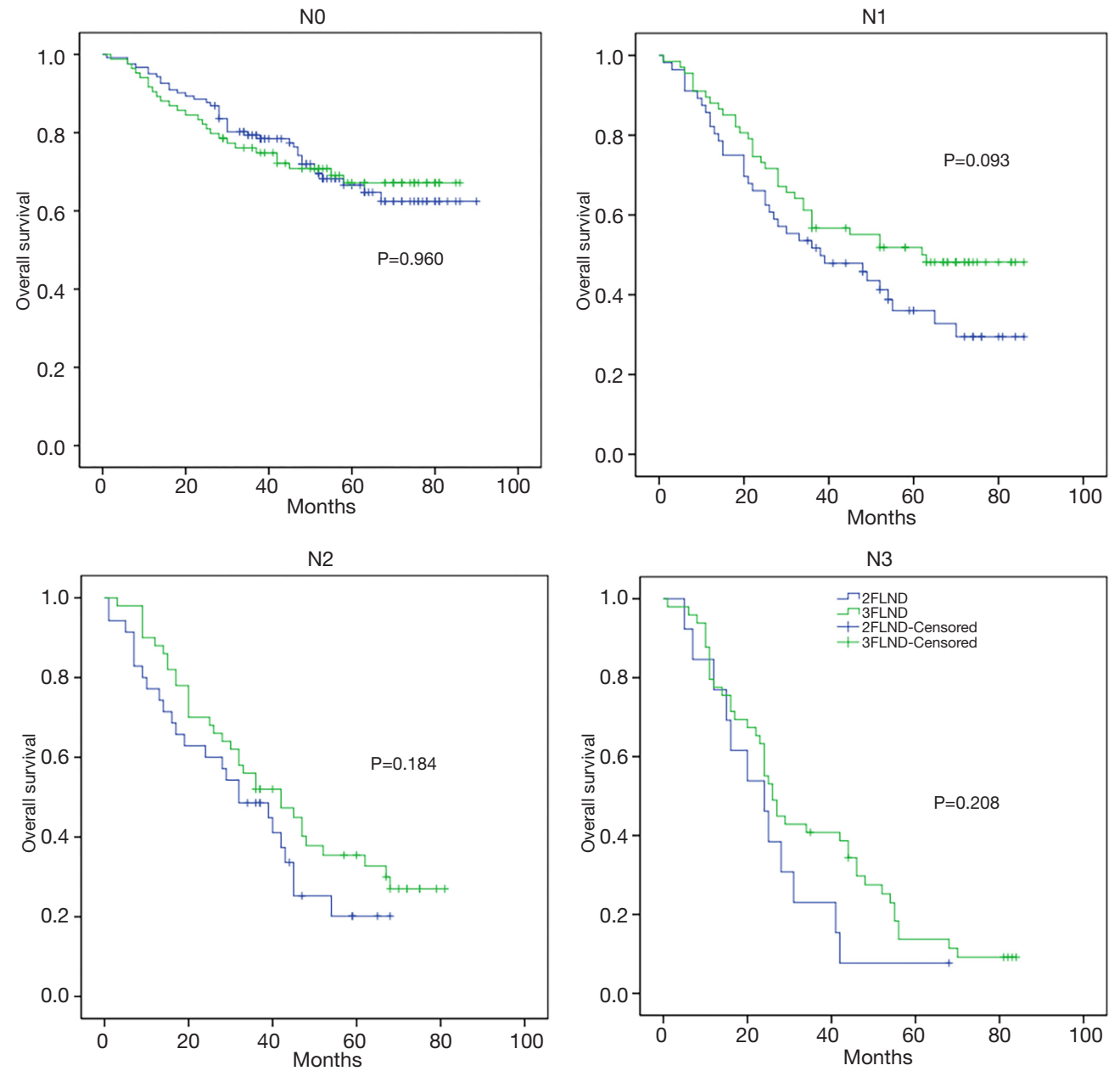

Figure 4 Kaplan-Meier curves for overall survival for each nodal stage. 2FLND, two-field lymph node dissection; 3FLND, three-field lymph node dissection.

Table 4 Factors of anastomotic leak

\begin{tabular}{|c|c|c|c|c|c|}
\hline Variable & Total/leak & \multicolumn{2}{|c|}{ Univariate } & \multicolumn{2}{|c|}{ Multivariate } \\
\hline \multicolumn{6}{|c|}{ Hand or mechanical } \\
\hline Mechanical & $500 / 42$ & & & & \\
\hline Hand & $64 / 12$ & $2.516(1.246-5.081)$ & 0.008 & $2.000(0.905-4.417)$ & 0.086 \\
\hline 2FLND & $282 / 12$ & & & & \\
\hline 3FLND & $282 / 42$ & $3.938(2.026-7.654)$ & $<0.001$ & $0.993(0.365-2.702)$ & 0.988 \\
\hline \multicolumn{6}{|c|}{ Anastomotic location } \\
\hline Neck & $140 / 36$ & & & & \\
\hline
\end{tabular}

2FLND, two-field lymph node dissection; 3FLND, three-field lymph node dissection. 


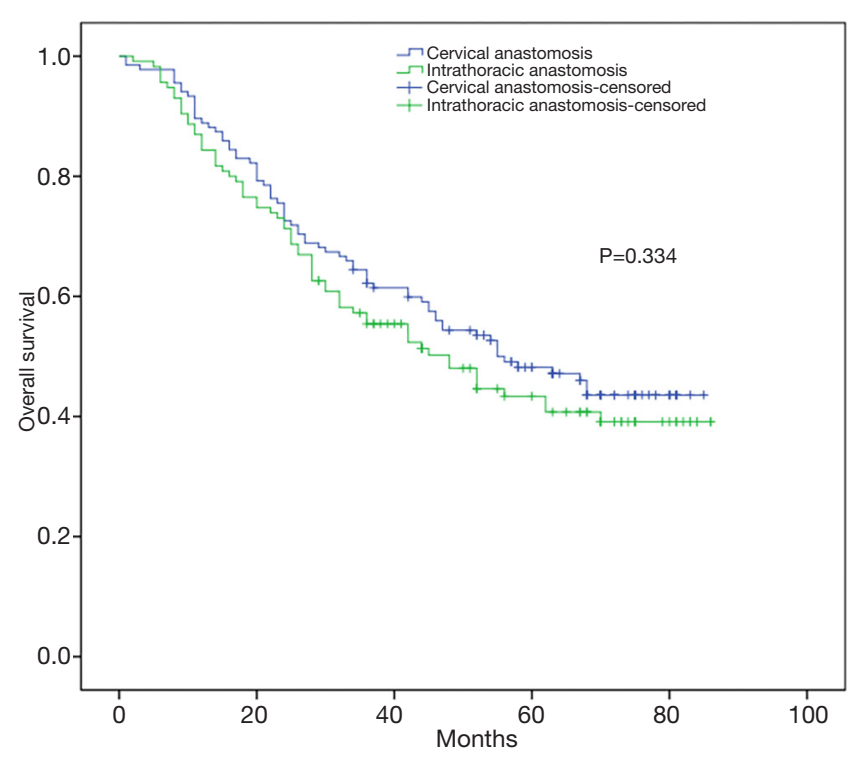

Figure 5 Overall survival according to anastomosis location in 3FLND. 3FLND, three-field lymph node dissection.

study found no difference in overall survival between the 3FLND and 2FLND groups. We performed 3FLND in select patients, most of whom had clinical cervical or upper mediastinum lymph node positivity. A limitation of our study is that there was still a bias despite propensity matching. The number of metastatic lymph nodes in the 3 FLND group was more than that in the 2FLND group (3.5 vs. 1.6, $\mathrm{P}<0.001)$. This may have a negative impact on the survival of 3FLND group. Our subgroup analysis showed that patients with the N1 status receiving 3FLND experienced a survival benefit trend. For these patients, 3FLND may be a better choice. The number of lymph node metastases of the N1 status in the two groups was more consistent than other status, and for those patients, the 3FLND technique may be a more curative treatment. However, further studies are needed to confirm this. The extent of lymphadenectomy for thoracic esophageal cancer remains controversial because of the paucity of largesample studies. Patients in this study were from a single, high-volume institute, admitted within a short period. This research design may provide more convincing evidence of these two procedures in current stage. Our center is conducting a prospective randomized controlled study on comparing 3FLND and 2FLND for esophageal cancer. The enrollment for this study has been completed, and the results will be reported in the future.

\section{Conclusions}

Compared to 2FLND, the incidence of postoperative complications especially anastomotic leakage is higher in 3FLND. A Change of anastomosis location, from the neck to the chest apex, can reduce the incidence of anastomotic leakage. 3FLND may provide a better overall survival benefit for patients with N1 status, but not for all patients from our experience.

\section{Acknowledgements}

None.

\section{Footnote}

Conflicts of Interest: The authors have no conflicts of interest to declare

Ethical Statement: The database analyzed for this study was approved for use by the institutional review board of Fudan University Shanghai Cancer Center (ID: 1211116-1). Clinical data from the patients were reviewed retrospectively.

\section{References}

1. Akiyama H, Tsurumaru M, Udagawa H, et al. Radical lymph node dissection for cancer of the thoracic esophagus. Ann Surg 1994;220:364-72; discussion 372-3.

2. Udagawa $H$, Ueno $M$, Shinohara $H$, et al. The importance of grouping of lymph node stations and rationale of threefield lymphoadenectomy for thoracic esophageal cancer. J Surg Oncol 2012;106:742-7.

3. Ye T, Sun Y, Zhang Y, et al. Three-field or two-field resection for thoracic esophageal cancer: a meta-analysis. Ann Thorac Surg 2013;96:1933-41

4. Li H, Yang S, Zhang Y, et al. Thoracic recurrent laryngeal lymph node metastases predict cervical node metastases and benefit from three-field dissection in selected patients with thoracic esophageal squamous cell carcinoma. J Surg Oncol 2012;105:548-52.

5. Li H, Zhang Y, Cai H, et al. Pattern of lymph node metastases in patients with squamous cell carcinoma of the thoracic esophagus who underwent three-field lymphadenectomy. Eur Surg Res 2007;39:1-6.

6. Kato H, Tachimori Y, Watanabe H, I et al. Lymph node metastasis in thoracic esophageal carcinoma. J Surg Oncol 
1991;48:106-11.

7. Lerut T, Nafteux P, Moons J, et al. Three-field lymphadenectomy for carcinoma of the esophagus and gastroesophageal junction in $174 \mathrm{R} 0$ resections: impact on staging, disease-free survival, and outcome: a plea for adaptation of TNM classification in upper-half esophageal carcinoma. Ann Surg 2004;240:962-72; discussion 972-4.

8. Altorki N, Kent M, Ferrara C, et al. Three-field lymph node dissection for squamous cell and adenocarcinoma of the esophagus. Ann Surg 2002;236:177-83.

9. Junemann-Ramirez M, Awan MY, Khan ZM, et al. Anastomotic leakage post-esophagogastrectomy for esophageal carcinoma: retrospective analysis of predictive factors, management and influence on longterm survival in a high volume centre. Eur J Cardiothorac Surg 2005;27:3-7.

10. Karl RC, Schreiber R, Boulware D, et al. Factors affecting morbidity, mortality, and survival in patients undergoing Ivor Lewis esophagogastrectomy. Ann Surg 2000;231:635-43.

11. Li B, Xiang J, Zhang Y, et al. Factors Affecting Hospital Mortality in Patients with Esophagogastric Anastomotic Leak: A Retrospective Study. World J Surg 2016;40:1152-7.

12. Ma GW, Situ DR, Ma QL, et al. Three-field vs two-field lymph node dissection for esophageal cancer: a metaanalysis. World J Gastroenterol 2014;20:18022-30.
Cite this article as: Shao L, Ye T, Ma L, Lin D, Hu H, Sun Y, Zhang Y, Xiang J, Chen H. Three-field versus two-field lymph node dissection for thoracic esophageal squamous cell carcinoma: a propensity score-matched comparison. J Thorac Dis 2018;10(5):2924-2932. doi: 10.21037/jtd.2018.05.69 